\title{
JOINT SIGNAL PARAMETER ESTIMATION IN NON-GAUSSIAN NOISE BY THE METHOD OF POLYNOMIAL MAXIMIZATION
}

\author{
Volodymyr Palahin ${ }^{*}$ - Jozef Juhár ${ }^{* *}$
}

\begin{abstract}
This paper considers the adaptation of the method of polynomial maximization for synthesis of the polynomial algorithms of joint signal parameter estimation in non-Gaussian noise. It is shown that the nonlinear processing of samples, the moment and the cumulant description of random variables in the form of cumulant coefficients of the third and higher orders can decrease the variance of joint parameters estimation as compared with the well-known results.
\end{abstract}

K e y w ords: the method of polynomial maximization, joint parameter estimation, non-Gaussian noise

\section{INTRODUCTION}

Traditionally, the design of the parameter estimation systems is based on the classical signal processing methods where the normal (Gaussian) probability density function (PDF) of the random processes is usually used [1-3]. Gaussian PDF of the stochastic processes is widely applied, but in many cases it does not describe the real processes with the desired accuracy and turns out to be a convenient mathematical idealization of the real stochastic process [4-7]. The traditional approaches are characterized by significant limitations of the parameter estimation of the non-Gaussian stochastic processes. Such problems are associated with the complexity of their algorithmic implementation and the increasing of the computer resources. In order to solve these problems, we can use another approach which is based on higher-order statistics (HOS). According to this approach, the random processes can be described in the form of the moment and cumulant (semivariants) functions. Such functions describe the statistical properties of the non-Gaussian processes with a reasonable accuracy [8-11]. Partial description of the random processes in the form of moments and cumulants finite sequence allows us to apply more effective processing of the non-Gaussian processes [10-14]. Another approach, introduced on the basis of the moment and cumulant description of the random variables, allows us to apply the Method of Polynomial Maximization (MPM) and to get the asymptotically-efficient parameter estimation of non-Gaussian random variables $[10,11]$. The effective polynomial algorithms for scalar parameter estimation of the non-Gaussian random processes have been synthesized on the basis of the MPM [12-14]. However, in many practical cases, the joint parameter estimation is more important and widely used for the synthesis of the signal processing systems $[15,16]$. The adaptation of the MPM for the joint parameter estimation for unequally distributed random values in non-Gaussian noise is suggested.

The main objective of the paper is the synthesis and the analysis of the method of the joint radiofrequency signal parameter estimation in non-Gaussian noise. The basis of this method is the moment and the cumulant description of the random variables and a stochastic power polynomial for synthesis of the effective algorithms and the computer tools for the function of data processing systems.

\section{ADAPTATION THE METHOD OF POLYNOMIAL MAXIMIZATION FOR JOINT PARAMETER ESTIMATION}

Let the random signal $\xi(t)$ be observed in the time interval $[0, T]$ and consist of the useful radiofrequency signal $S(t)$ and noise $\eta(t)$

$$
\xi(t)=S(t)+\eta(t),
$$

where $\eta(t)$ - non-Gaussian stationary stochastic process that describes the sequence of moments $\alpha_{i}$ and cumulants $\chi_{i}$ with a zero meaning, variance $\chi_{2}$ and the cumulant coefficients $\gamma_{i}\left(\gamma_{i}=\chi_{i} / \chi_{2}^{i / 2}\right)$ are not equal to zero $\left(\gamma_{i} \neq 0\right.$, $i \geq 3)$ [9-11], $S(t)=A e(t) \cos (\omega t+\varphi)$ - radiofrequency signal with the unknown parameters $\boldsymbol{\vartheta}=\{A, \omega, \varphi\}$, such as the amplitude $A$, frequency $\omega$ and phase $\varphi$.

If the sampling signal is $\xi(t)$, we get their discrete independent values $\mathbf{X}=\left\{x_{1}, x_{2}, \ldots, x_{n}\right\}$ in the time $t_{v}$ and the input signal is defined as

$$
x_{v}=S_{v(\boldsymbol{\vartheta})}+\eta_{v}, \quad v=1, \ldots, n,
$$

where $S_{v(\boldsymbol{\vartheta})}=A e_{v} \cos \left(\omega \Delta_{v}+\varphi\right), \Delta_{v}$ - the step of sampling.

\footnotetext{
* Department of Radio Engineering, Information and Telecommunication Systems, Cherkasy State Technological University, Shevchenko blvd. 460, 18006 Cherkasy, Ukraine, Palahin@yahoo.com; ${ }^{* *}$ Department of Electronics and Multimedia Communications, Technical University of Košice, Park Komenckého 13, 04120 Košice, Slovak Republic, Jozef.Juhar@gmail.com
} 
Then initial moments of $\xi(t)$ are defined as $m_{i(v)}(\boldsymbol{\vartheta})=$ $E\left(\xi_{v}\right)^{i}=E\left(S_{v(\vartheta)}+\eta\right)^{i}$.

The problem is the vector $\boldsymbol{\vartheta}=\{A, \omega, \varphi\}$ parameter estimation of the random non-Gaussian process (1). We examine the adaptation of the MPM for synthesis of the algorithms joint radiofrequency signal parameter estimation in non-Gaussian noise.

According to the MPM, the joint radiofrequency parameter estimation $\boldsymbol{\vartheta}=\{A, \omega, \varphi\}$ can be found from solution of the equations of the power polynomial maximization of the degree $s$ for each component $\vartheta_{k}$, where the additional sum for each component of sample $x_{v}$ will be used:

$$
\begin{array}{r}
\left.\sum_{i=1}^{s} \sum_{v=1}^{n} h_{i(k) v[s]}(\boldsymbol{\vartheta})\left(x_{v}^{i}-m_{i(v)}(\boldsymbol{\vartheta})\right)\right|_{S_{v(\boldsymbol{\vartheta})}=\hat{S}_{v(\vartheta)}}=0, \\
k=1,2,3,
\end{array}
$$

where $h_{i(k)(v)[s]}(\boldsymbol{\vartheta})$ - unknown coefficients which are found from the system of linear equations for each component $k$ :

$$
\begin{aligned}
\sum_{j=1}^{s} h_{j(k) v[s]}(\boldsymbol{\vartheta}) K_{i, j(v)}(\boldsymbol{\vartheta}) & =\frac{\mathrm{d}}{\mathrm{d} \vartheta_{k}} m_{i(v)}(\boldsymbol{\vartheta}), \\
i=1, \ldots, s, & v=1, \ldots, n, k=1,2,3,
\end{aligned}
$$

where $K_{i, j(v)}(\boldsymbol{\vartheta})=m_{(i+j)(v)}(\boldsymbol{\vartheta})-m_{i(v)}(\boldsymbol{\vartheta}) m_{j(v)}(\boldsymbol{\vartheta})$.

The matrix of the derived information quantity [10] from the samples of the volume $\mathrm{n}$ is used to obtain the variances of vector parameters estimation by the adapted MPM (AMPM):

$$
J_{s n}(\boldsymbol{\vartheta})=\left(\begin{array}{lll}
J_{s n}^{(1,1)}(\boldsymbol{\vartheta}) & J_{s n}^{(1,2)}(\boldsymbol{\vartheta}) & J_{s n}^{(1,3)}(\boldsymbol{\vartheta}) \\
J_{s n}^{(2,1)}(\boldsymbol{\vartheta}) & J_{s n}^{(2,2)}(\boldsymbol{\vartheta}) & J_{s n}^{(2,3)}(\boldsymbol{\vartheta}) \\
J_{s n}^{(3,1)}(\boldsymbol{\vartheta}) & J_{s n}^{(3,2)}(\boldsymbol{\vartheta}) & J_{s n}^{(3,3)}(\boldsymbol{\vartheta})
\end{array}\right)
$$

where

$$
\begin{aligned}
& J_{s n}^{(1,1)}(\boldsymbol{\vartheta})=\sum_{i=1}^{s} \sum_{v=1}^{n} h_{i(1) v[i]}(\boldsymbol{\vartheta}) \frac{\mathrm{d} m_{i(v)}(\boldsymbol{\vartheta})}{\mathrm{d} A}, \\
& J_{s n}^{(2,2)}(\boldsymbol{\vartheta})=\sum_{i=1}^{s} \sum_{v=1}^{n} h_{i(2) v[i]}(\boldsymbol{\vartheta}) \frac{\mathrm{d} m_{i(v)}(\boldsymbol{\vartheta})}{\mathrm{d} \omega}, \\
& J_{s n}^{(1,2)}(\boldsymbol{\vartheta})=J_{s n}^{(2,1)}(\boldsymbol{\vartheta})=\sum_{i=1}^{s} \sum_{v=1}^{n} h_{i(1) v[i]}(\boldsymbol{\vartheta}) \frac{\mathrm{d} m_{i(v)}(\boldsymbol{\vartheta})}{\mathrm{d} \omega} \\
& =\sum_{i=1}^{s} \sum_{v=1}^{n} h_{i(2) v[i]}(\boldsymbol{\vartheta}) \frac{\mathrm{d} m_{i(v)}(\boldsymbol{\vartheta})}{\mathrm{d} A}, \\
& J_{s n}^{(1,3)}(\boldsymbol{\vartheta})=J_{s n}^{(3,1)}(\boldsymbol{\vartheta})=\sum_{i=1}^{s} \sum_{v=1}^{n} h_{i(1) v[i]}(\boldsymbol{\vartheta}) \frac{\mathrm{d} m_{i(v)}(\boldsymbol{\vartheta})}{\mathrm{d} \boldsymbol{\vartheta}} \\
& =\sum_{i=1}^{s} \sum_{v=1}^{n} h_{i(3) v[i]}(\boldsymbol{\vartheta}) \frac{\mathrm{d} m_{i(v)}(\boldsymbol{\vartheta})}{\mathrm{d} A}, \\
& J_{s n}^{(2,3)}(\boldsymbol{\vartheta})=J_{s n}^{(3,2)}(\boldsymbol{\vartheta})=\sum_{i=1}^{s} \sum_{v=1}^{n} h_{i(3) v[i]}(\boldsymbol{\vartheta}) \frac{\mathrm{d} m_{i(v)}(\boldsymbol{\vartheta})}{\mathrm{d} \omega} \\
& J_{s n}^{(3,3)}(\boldsymbol{\vartheta})=\sum_{i=1}^{s} \sum_{v=1}^{n} h_{i(3) v[i]}(\boldsymbol{\vartheta}) \frac{\mathrm{d} m_{i(v)}(\boldsymbol{\vartheta})}{\mathrm{d} \varphi} .
\end{aligned}
$$

The $J_{s n}^{(r, r)}(\boldsymbol{\vartheta}), r=1,2,3$ is the derived information quantity about $r$-th parameter for separate scalar parameter estimation, at that $J_{s n}^{(k, z)}(\boldsymbol{\vartheta})=J_{s n}^{(z, k)}(\boldsymbol{\vartheta})$.

In this case the variances of joint $\boldsymbol{\vartheta}=\{\hat{A}, \hat{\omega}, \hat{\varphi}\}$ parameters estimation will be accordingly equal to the diagonal elements of the variation matrix and asymptotically for $n \rightarrow \infty$ is equal to the matrix of the information quantity $J_{s n}(\boldsymbol{\vartheta})$ :

$$
\begin{aligned}
& \sigma_{(A)[s]}^{2}(\boldsymbol{\vartheta})=\frac{J_{s n}^{(2,2)}(\boldsymbol{\vartheta}) J_{s n}^{(3,3)}(\boldsymbol{\vartheta})-\left(J_{s n}^{(2,3)}(\boldsymbol{\vartheta})\right)^{2}}{\left\|J_{s n}(\boldsymbol{\vartheta})\right\|}, \\
& \sigma_{(\omega)[s]}^{2}(\boldsymbol{\vartheta})=\frac{J_{s n}^{(1,1)}(\boldsymbol{\vartheta}) J_{s n}^{(3,3)}(\boldsymbol{\vartheta})-\left(J_{s n}^{(1,3)}(\boldsymbol{\vartheta})\right)^{2}}{\left\|J_{s n}(\boldsymbol{\vartheta})\right\|}, \\
& \sigma_{(\varphi)[s]}^{2}(\boldsymbol{\vartheta})=\frac{J_{s n}^{(1,1)}(\boldsymbol{\vartheta}) J_{s n}^{(2,2)}(\boldsymbol{\vartheta})-\left(J_{s n}^{(1,2)}(\boldsymbol{\vartheta})\right)^{2}}{\left\|J_{s n}(\boldsymbol{\vartheta})\right\|} .
\end{aligned}
$$

The variance decreasing coefficient is used for assess of the efficiency of the variance joint parameters estimation of $\boldsymbol{\vartheta}=\{\hat{A}, \hat{\omega}, \hat{\varphi}\}$ :

$$
g_{(k) s r}(\vartheta)=\frac{\sigma_{(k)[s]}^{2}(\boldsymbol{\vartheta})}{\sigma_{(k)[r]}^{2}(\boldsymbol{\vartheta})},
$$

where $\sigma_{(k)[s]}^{2}(\boldsymbol{\vartheta})$ - the variance of the component $\vartheta_{k}$ estimation of the vectors parameter $\theta$ that was found by AMPM for degree $s, \sigma_{(k)[r]}^{2}(\boldsymbol{\vartheta})$ - variance of the component $\vartheta_{k}$ estimation of vectors parameter $\boldsymbol{\vartheta}$ that was found by AMPM for degree $r$.

Let us synthesize the polynomial algorithms of joint parameter estimation and analyze their effectiveness by the AMPM.

\section{THE SYNTHESIS OF THE POLYNOMIAL ALGORITHMS OF JOINT PARAMETER ESTIMATION}

Let us consider a non-Gaussian process that is described by a sequence of moments and cumulants. As an example, let $\eta(t)$ - non-Gaussian stationary asymmetrical stochastic process (which is characterized by zero mean, $\chi_{2}$ variance and $\gamma_{3}$ asymmetry coefficient) be not equal to zero $\left(\gamma_{4}=0, \gamma_{i}-\right.$ not determined for $\left.i>4\right)$.

Let us assume that $\chi_{2}$ and $\gamma_{3}$ are known and can be written down as $\chi_{20}, \gamma_{30}$ accordingly. Then the initial moments $\alpha_{i}\left(\chi_{20}, \gamma_{30}\right)$ of $\eta(t)$ as the fourth degree are defined

$$
\alpha_{1}(\cdot)=0, \alpha_{2}(\cdot)=\chi_{20}, \alpha_{3}(\cdot)=\chi_{20}^{1.5} \gamma_{30}, \alpha_{4}(\cdot)=3 \chi_{20}^{2}
$$

and the initial moments $m_{i(v)}(\boldsymbol{\vartheta})$ of $\xi(t)$ to four degree look like

$$
\begin{aligned}
& m_{1(v)}(\boldsymbol{\vartheta})=S_{v(\boldsymbol{\vartheta})}, \quad m_{2(v)}(\boldsymbol{\vartheta})=S_{v(\boldsymbol{\vartheta})}^{2}+\chi_{20}, \\
& m_{3(v)}(\boldsymbol{\vartheta})=S_{v(\boldsymbol{\vartheta})}^{3}+3 S_{v(\boldsymbol{\vartheta})} \chi_{20}+\gamma_{30} \chi_{20}^{1.5}, \\
& m_{4(v)}(\boldsymbol{\vartheta})=S_{v(\boldsymbol{\vartheta})}^{4}+6 S_{v(\boldsymbol{\vartheta})}^{2} \chi_{20}+4 S_{v(\boldsymbol{\vartheta})} \gamma_{30} \chi_{20}^{1.5}+3 \chi_{20}^{2} .
\end{aligned}
$$

The unknown coefficient $K_{i, j(v)}(\boldsymbol{\vartheta})(3)$ is defined for such asymmetric non-Gaussian model of random process in the following way.

$$
\begin{aligned}
& K_{1,1(v)}(\boldsymbol{\vartheta})=\chi_{20}, \\
& K_{1,2(v)}(\boldsymbol{\vartheta})=K_{2,1(v)}(\boldsymbol{\vartheta})=\chi_{20}\left[2 S_{v(\boldsymbol{\vartheta})}+\gamma_{30} \chi_{2}^{0.5}\right], \\
& K_{2,2(v)}(\boldsymbol{\vartheta})=2 \chi_{20}\left[2 S_{v(\boldsymbol{\vartheta})}^{2}+2 S_{v(\boldsymbol{\vartheta})} \gamma_{30} \chi_{20}^{0.5}+\chi_{20}\right] .
\end{aligned}
$$


Let us find the parameters $\vartheta$ for polynomial degree $s=1$ from (2) by the AMPM. Then the vector $\boldsymbol{\vartheta}$ elements estimation are found from the system of the three equations (2) for $\vartheta_{k}$ components:

$$
\left.\sum_{v=1}^{n} h_{1(k) v[1]}(\boldsymbol{\vartheta})\left(x_{v}-m_{1(v)}(\boldsymbol{\vartheta})\right)\right|_{\vartheta_{k}=\hat{\vartheta}_{k}}=0, \quad k=1,2,3,
$$

where the optimal coefficients are found for each of components from solution the system of equations (3):

$h_{1(k) v[1]}(\boldsymbol{\vartheta}) K_{1,1(v)}(\boldsymbol{\vartheta})=\frac{\mathrm{d} m_{1(v)}(\boldsymbol{\vartheta})}{\mathrm{d} \vartheta_{k}}, v=1, \ldots, n, k=1,2,3$.

The system of equations is defined from (7) for find the unknown parameters $\boldsymbol{\vartheta}$ of the stochastic power polynomial of degree $s=1$

$$
\begin{gathered}
\left.\sum_{v=1}^{n}\left(x_{v}-A e_{v} \cos \left(\omega \Delta_{v}+\varphi\right)\right) e_{v} \cos \left(\omega \Delta_{v}+\varphi\right)\right|_{A=\hat{A}}=0 \\
\left.\sum_{v=1}^{n}\left(x_{v}-A e_{v} \cos \left(\omega \Delta_{v}+\varphi\right)\right) \Delta_{v} e_{v} \sin \left(\omega \Delta_{v}+\varphi\right)\right|_{\omega=\hat{\omega}}=0 \\
\left.\sum_{v=1}^{n}\left(x_{v}-A e_{v} \cos \left(\omega \Delta_{v}+\varphi\right)\right) e_{v} \sin \left(\omega \Delta_{v}+\varphi\right)\right|_{\varphi=\hat{\varphi}}=0
\end{gathered}
$$

and is solved by the numerical methods.

Let us find the joint signal parameter estimation of the power polynomial degree $s=2$. The vector elements estimation are found from the system of the three equations (2) for $\vartheta_{k}$ components and is defined as

$$
\begin{aligned}
& \sum_{v=1}^{n} h_{1(k) v[2]}(\boldsymbol{\vartheta})\left(x_{v}-m_{1(v)}(\boldsymbol{\vartheta})\right)+ \\
& \left.h_{2(k) v[2]}(\boldsymbol{\vartheta})\left(x_{v}^{2}-m_{2(v)}(\boldsymbol{\vartheta})\right)\right|_{\vartheta_{k}=\hat{\vartheta}_{k}}=0, k=1,2,3,
\end{aligned}
$$

where the optimal coefficients $h_{1(k)(v)[2]}(\boldsymbol{\vartheta})$ and $h_{2(k)(v)[2]}(\boldsymbol{\vartheta})$ are defined for each components of $\vartheta_{k}$ from solution the system of equations (3):

$$
\begin{aligned}
& h_{1(k) v[2]\{s 11\}}(\boldsymbol{\vartheta}) K_{1,1(v)\{s 11\}}(\boldsymbol{\vartheta})+ \\
& h_{2(k) v[2]\{s 11\}}(\boldsymbol{\vartheta}) K_{1,2(v)\{s 11\}}(\boldsymbol{\vartheta})=\frac{\mathrm{d} m_{1(v)\{s 11\}}(\boldsymbol{\vartheta})}{\mathrm{d} \vartheta_{k}}, \\
& h_{1(k) v[2]\{s 11\}}(\boldsymbol{\vartheta}) K_{1,2(v)\{s 11\}}(\boldsymbol{\vartheta})+ \\
& h_{2(k) v[2]\{s 11\}}(\boldsymbol{\vartheta}) K_{2,2(v)\{s 11\}}(\boldsymbol{\vartheta})=\frac{\mathrm{d} m_{2(v)\{s 11\}}(\boldsymbol{\vartheta})}{\mathrm{d} \vartheta_{k}} .
\end{aligned}
$$

The system of equations is obtained from (8) to find the unknown parameters $\boldsymbol{\vartheta}$ of the stochastic power polynomial of the degree $s=2$ :

$$
\begin{gathered}
\sum_{v=1}^{n} e_{v} \cos \left(\omega \Delta_{v}+\varphi\right)\left[A^{2} e_{v}^{2} \cos ^{2}\left(\omega \Delta_{v}+\varphi\right) \gamma_{30}+\right. \\
2 A e_{v} \cos \left(\omega \Delta_{v}+\varphi\right)\left(-x_{v} \gamma_{30}+\chi_{20}^{0.5}\right)+ \\
\left.x_{v}^{2} \gamma_{30}-2 x_{v} \chi_{20}^{0.5}-\gamma_{30} \chi_{20}\right]\left.\right|_{A=\hat{A}}=0
\end{gathered}
$$

$$
\begin{gathered}
\sum_{v=1}^{n}\left[0.5 A^{3} \Delta_{v} e_{v}^{3} \gamma_{30} \cos \left(\omega \Delta_{v}+\varphi\right) \sin 2\left(\omega \Delta_{v}+\varphi\right)-\right. \\
A^{2} \Delta_{v} e_{v}^{2}\left(x_{v} \gamma_{30}-\chi_{20}^{0.5}\right) \sin 2\left(\omega \Delta_{v}+\varphi\right)+ \\
\left.A e_{v} \Delta_{v} \sin \left(\omega \Delta_{v}+\varphi\right)\left(x_{v}^{2} \gamma_{30}-2 x_{v} \chi_{20}^{0.5}-\gamma_{30} \chi_{20}\right)\right]\left.\right|_{\omega=\hat{\omega}}=0,
\end{gathered}
$$$$
\sum_{v=1}^{n}\left[0,5 A^{3} e_{v}^{3} \gamma_{30} \cos \left(\omega \Delta_{v}+\varphi\right) \sin 2\left(\omega \Delta_{v}+\varphi\right)-\right.
$$$$
A^{2} e_{v}^{2}\left(x_{v} \gamma_{30}-\chi_{20}^{0.5}\right) \sin 2\left(\omega \Delta_{v}+\varphi\right)+
$$$$
\left.A \sin \left(\omega \Delta_{v}+\varphi\right) e_{v}\left(x_{v}^{2} \gamma_{30}-2 x_{v} \chi_{20}^{0.5}-\gamma_{30} \chi_{20}\right)\right]\left.\right|_{\varphi=\hat{\varphi}}=0 \text {. }
$$

and is solved by the numerical methods.

The efficiency of the polynomial processing of the random variable by AMPM was carried out to compare the variances of the joint parameter estimation for the power polynomial of the degree $s=1,2$. The variances of the parameter estimation $(\hat{A}, \hat{\omega}$ and $\hat{\varphi})$ for $s=1$ are defined from (4-5) and look like

$$
\begin{gathered}
\sigma_{(A)[1]}^{2}(\boldsymbol{\vartheta})=\frac{A^{4}\left[\sum_{v=1}^{n} \Delta_{v}^{2} a_{v}^{2}-\left(\sum_{v=1}^{n} \Delta_{v} a_{v}\right)^{2}\right]}{\chi_{20}^{2}\left\|J_{1 n}(\boldsymbol{\vartheta})\right\|}, \\
\sigma_{(\omega)[1]}^{2}(\boldsymbol{\vartheta})=\frac{A^{2}\left[\sum_{v=1}^{n} a_{v} b_{v}-\frac{1}{4}\left(\sum_{v=1}^{n} a_{v}\right)^{2}\right]}{\chi_{20}^{2}\left\|J_{1 n}(\boldsymbol{\vartheta})\right\|}, \\
\sigma_{(\phi)[1]}^{2}(\boldsymbol{\vartheta})=\frac{A^{2}\left[\sum_{v=1}^{n} \Delta_{v}^{2} a_{v} b_{v}-\frac{1}{4}\left(\sum_{v=1}^{n} \Delta_{v} c_{v}\right)^{2}\right]}{\chi_{20}^{2}\left\|J_{1 n}(\boldsymbol{\vartheta})\right\|},
\end{gathered}
$$

where

$$
\begin{gathered}
\left\|J_{1 n}(\boldsymbol{\vartheta})\right\|=\frac{-A^{4}}{4 \chi_{20}^{1.5}}\left[-2 \sum_{v=1}^{n} \Delta_{v}^{2} d_{v}^{2} a_{v}+\sum_{v=1}^{n} a_{v}\left(\sum_{v=1}^{n} \Delta_{v} d_{v}\right)^{2}+\right. \\
\left.\sum_{v=1}^{n} \Delta_{v}^{2} a_{v}\left(\sum_{v=1}^{n} d_{v}\right)^{2}+4 \sum_{v=1}^{n} b_{v}\left[\left(\sum_{v=1}^{n} \Delta_{v} a_{v}\right)^{2}-\sum_{v=1}^{n} \Delta_{v}^{2} a_{v}^{2}\right]\right] \\
a_{v}=e_{v}^{2} \sin ^{2}\left(\omega \Delta_{V}+\varphi\right), \quad b_{v}=e_{v}^{2} \cos ^{2}\left(\omega \Delta_{V}+\varphi\right) \\
c_{v}=e_{v}^{2} \sin ^{2}\left(2 \omega \Delta_{V}+2 \varphi\right), \quad d_{v}=e_{v}^{2} \sin \left(2 \omega \Delta_{V}+2 \varphi\right) .
\end{gathered}
$$

It can be noticed that the variance values of the joint parameter estimation for $s=1$ are the same as when the well-known method of maximum likelihood estimation (MLE) for the Gaussian PDF is used. However, these variance values differ from the mentioned above in the power polynomial of the degree $s=2$ and are defined as

$$
\begin{aligned}
\sigma_{(A)[2]}^{2}(\boldsymbol{\vartheta}) & =\frac{4 A^{4}\left[\sum_{v=1}^{n} \Delta_{v}^{2} a_{v}^{2}-\left(\sum_{v=1}^{n} \Delta_{v} a_{v}\right)^{2}\right]}{\chi_{20}^{2}\left(2-\gamma_{30}^{2}\right)^{2}\left\|J_{2 n}(\boldsymbol{\vartheta})\right\|}, \\
\sigma_{(\omega)[2]}^{2}(\boldsymbol{\vartheta}) & =\frac{4 A^{2}\left[\sum_{v=1}^{n} a_{v} b_{v}-\frac{1}{4}\left(\sum_{v=1}^{n} a_{v}\right)^{2}\right]}{\chi_{20}^{2}\left(2-\gamma_{30}^{2}\right)^{2}\left\|J_{2 n}(\boldsymbol{\vartheta})\right\|}, \\
\sigma_{(\varphi)[2]}^{2}(\boldsymbol{\vartheta}) & =\frac{4 A^{2}\left[\sum_{v=1}^{n} \Delta_{v}^{2} a_{v} b_{v}-\frac{1}{4}\left(\sum_{v=1}^{n} \Delta_{v} c_{v}\right)^{2}\right]}{\chi_{20}^{2}\left(2-\gamma_{30}^{2}\right)^{2}\left\|J_{2 n}(\boldsymbol{\vartheta})\right\|},
\end{aligned}
$$



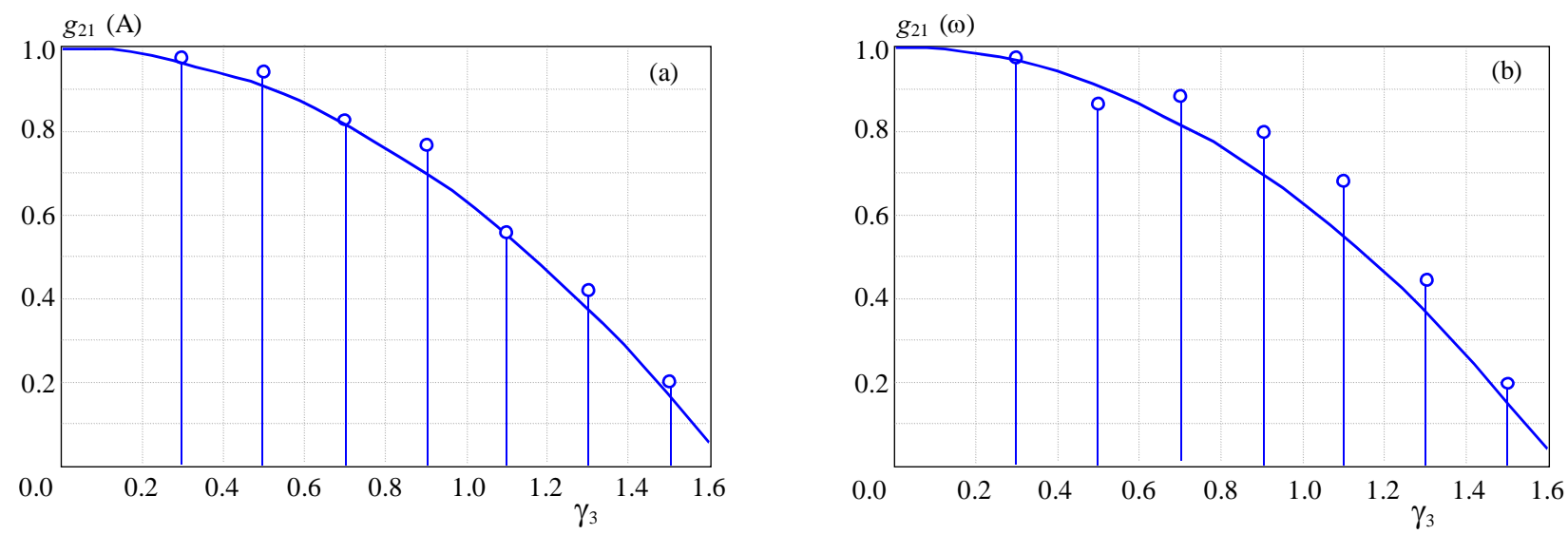

Fig. 1. The comparison of the theoretical (curve) and the experimental (dots) results of the VRC $g_{21}(\vartheta)$ of the joint parameter estimation, (a) - amplitude, (b) - frequency, for power the polynomial of the degree $s=1,2$ from the asymmetry coefficient $\gamma_{3}$

where $\left\|J_{2 n}(\boldsymbol{\vartheta})\right\|=\frac{8\left\|J_{1 n}(\boldsymbol{\vartheta})\right\|}{\left(2-\gamma_{30}^{2}\right)^{3}}$.

The variance reduction coefficients (VRC) were calculated from (6):

$$
g_{(A) 21}(\vartheta)=g_{(\omega) 21}(\vartheta)=g_{(\varphi) 21}(\vartheta)=g_{21}(\vartheta)=1-\frac{\gamma_{30}^{2}}{2}
$$

where the tolerance range for asymmetry coefficient $\gamma_{3}$ is defined as $\gamma_{30}^{2} \leq \gamma_{40}+2[9,10]$ and the asymmetry coefficient looks like $\gamma_{30}^{2} \leq \sqrt{2}$ for asymmetric non-Gaussian noise model (then $\gamma_{40}=0$ ).

\section{RESULTS AND DISCUSSION}

This paper offers the joint parameter estimation of radiofrequency signals in the non-Gaussian noise on the basis of the AMPM. The effectiveness of estimation depends on the characteristics of the non-Gaussian random variables (9) (namely, from the asymmetry coefficient $\gamma_{3}$ ). It is shown that the variances of the joint signal parameter estimation are equal to each other for the power polynomial of the degree $s=1,2$ for $\gamma_{3}=0$ (Fig. 1). The variances of the joint parameter non-liner estimation are less for the power polynomial of the degree $s=2\left(\gamma_{3} \neq 0\right)$ than the variances of the linear estimation for the polynomial of the degree $s=1\left(\gamma_{3}=0\right)$. Besides, the linear estimation results for the polynomial of the degree $s=1$ are the same as the well-known results of the MLE method for Gaussian PDF.

It can be seen that the experimental (are marked as dots) and theoretical results (are marked as a curve) are approximately moved closer to each other. Taking into account the moments and the cumulants of the third and the higher order, for example, the asymmetry distribution of random samples (when $\gamma_{3} \neq 0$ ), this approach enables us to reduce the variances of the joint parameter estimation as compared with the well-known results. For example, the variance of the amplitude estimation is decreased approximately in $35 \%$ for $\gamma_{3}=1$ as compared with such parameter for the Gaussian model noise
(Fig. 1a). The variances of the joint parameter estimation will be less when the stochastic power polynomial of the degree $s$ increases.

\section{CONCLUSIONS}

The complex description of the non-Gaussian processes requires a new approach to solve the problems of parameter estimation. This approach is based on the utilizing of the new Method of Polynomial Maximization (MPM, Kunchenko method). We scrutinized the adaptation of the MPM for the joint parameter estimation for non-equal distributed samples. The effective algorithms of the joint radiofrequency signal parameter estimation in non-Gaussian are obtained. Taking into account the parameters of non-Gaussian distribution of random variables in form of the cumulant coefficients of the third and higher orders, this new method enables us to reduce the variances of the joint parameter estimation as compared with the well-known results.

\section{REFERENCES}

[1] KAY, S. M.: Fundamentals of Statistical Signal Processing: Estimation theory, Prentice-Hall, New Jersey, 1993.

[2] VAN TREES, H. L.: Detection, Estimation, and Modulation Theory. Part IV: Optimum Array Processing, John Wiley, 2002.

[3] TUZLUKOV, V. P.: Signal Processing Noise, CRC Press LLC, 2002.

[4] BARKAT, M. : Signal Detection and Estimation, Artech House, 2005.

[5] MIDDLETON, D.: Non-Gaussian Statistical Communication Theory, Jonn Willey \& Sons, 2012.

[6] KASSAM, S.: Signal Detection in Non-Gaussian Noise, Springer Verlag, 1988.

[7] TA-HSIN LI'-KAI-SHENG SONG : Estimation of The Parameters of Sinusoidal Signals in Non-Gaussian Noise, IEEE Transactions on Signal Processing 57 No. 1 (2009), 62-72.

[8] PAWI, A.-VASEGHI, S. : Fundamental Frequency Estimation Using Modified Higher Order Moments and Multiple Windows, Conference: Interspeech 2011, 12 th Annual Conference of the International Speech Communication Association, Florence, Italy, August 27-31, 2011. 
[9] MALAKHOV, A.: Cumulant Analysis of Non-Gaussian Processes and their Transformation, Sov. radio, 1979.

[10] KUNCHENKO, Y.: Polynomial Parameter Estimations of Close to Gaussian Random Variables, Shaker Verlag, Aachen, 2002.

[11] KUnCHENKO, Y.: Stochastic Polynomials, Naukova Dumka, 2006.

[12] PAlAhin, V.-HONCHAROV, A.-UMANETS, V.: Polynomial Algorithms Joint Signals Discrimination and Estimation of Their Parameters in Asymmetric non-Gaussian Noise, International scientific-theoretical journal Electronic modeling No. 4 (2014), 51-67.

[13] PAlahin, V.-HONCharov, A.-UMAnETS, V.: Computer Simulation of the Polynomial Algorithms Radiofrequency Signal Discrimination and Estimation their Parameters, Eastern-European Journal of Enterprise Technologies 9(71) No. 5 (2014), 31-39.

[14] PALAHIN, V.-PALAHINA, O.-FILIPOV, V.-LELEKO, S.-IVCHENKO, A.: Modeling of Joint Signal Detection and Parameter Estimation on Background of Non-Gaussian Noise, Journal of Applied Mathematics and Computational Mechanics 14 No. 3 (2015), 87-94.

[15] OLMO, G.-MAGLI, E.-LO PRESTI, L.: Joint Statistical Signal Detection and Estimation. Part I: Theoretical Aspects of the Problem, Signal Processing 80 No. 1 (2000), 57-73.

[16] CHEN, T.-C.: Joint Signal Parameter Estimation of Frequency-Hopping Communications, Communications, IET No. 4 (2012), 381389 .
Received 17 February 2016

Volodymyr Palahin graduated from Kiev Polytechnic Institute (NTUU "KPI") in 1992. He received his PhD degree in radioengineering from the Odesa Nationality Polytechnical University (Ukraine) in 1999 and Dr.Sc. degree in mathematical modelling from Pukhov Institute for Modelling in Energy Engineering (Ukraine) in 2014. He works as an Head at the Department of Radio Engineering, Information and Telecommunication Systems (Cherkasy State Technological University, Ukraine). He is author and co-author of more than 100 scientific papers. His research interests include the integration of signal processing, theoretical statistics and mathematics in practical applications - signals detection and recognition, parameters estimation in electrical engineering.

Jozef Juhár graduated from the Technical University of Kosice in 1980. He received his $\mathrm{PhD}$ degree in radioelectronics from the same university in 1991, where he works as an Head at the Department of Electronics and Multimedia Communications. He is author and co-author of more than 100 scientific papers. His research interests include digital speech and audio processing, speech and speaker recognition, speech synthesis and spoken dialogue systems in telecommunication networks. 